\title{
¿ES SU MASCOTA SALUDABLE? UN ESTUDIO SOBRE LA ACEPTACIÓN DE LA COMIDA ORGÁNICA PARA MASCOTAS
}

IS YOUR PET HEALTHY? A STUDY ON THE ACCEPTANCE OF ORGANIC FOOD FOR PETS

\author{
Santiago Cano González $\cdot$ Lina M. Ceballos ${ }^{\mathrm{b}}$ \\ Clasificación: Trabajo empírico - investigación \\ Recibido: 4-febrero-2019 / Aceptado: 15-agosto-2019
}

\section{Resumen}

Cifras a nivel global indican que la comida para mascotas presenta un constante crecimiento, y esta tendencia proyecta ocupar un alto porcentaje del mercado de productos para animales para el año 2021. Ante la necesidad de una mayor investigación que aborde el vínculo mascota-humano desde la perspectiva del comportamiento del consumidor, así como el desconocimiento del mercado de comida orgánica para mascotas en Medellín (Colombia), esta investigación cuantitativa explora la aceptación que pueden tener estos productos en dicha ciudad. También busca conocer las actitudes e intención de compra de este tipo de comida y su relación con la humanización de mascotas. A partir de una encuesta realizada en línea se captaron 150 registros de consumidores entre los 18 y los 65 años, de los niveles socioeconómicos medio y alto $(4,5$ y 6$)$ de la ciudad de Medellín. Los hallazgos evidencian que son las mujeres quienes tienen actitudes más positivas hacia la comida orgánica para mascotas, entendiendo que actitudes más positivas hacia este tipo de alimentos se traducen en una mayor intención de compra. Respecto al nivel de humanización de las mascotas se encontró que no existen diferencias significativas entre los encuestados. Este estudio se posiciona y aporta dentro del entendimiento del mercado de este tipo de alimentos en la capital de Antioquia.

Palabras clave: mascotas saludables, humanización, relación hombre-animal, comida orgánica, comida natural, actitudes.

\begin{abstract}
Global market data indicates that pet food is on the rise, with a projection to occupy a high percentage of the market for animal products by the year 2021. Given the need for more research related to the pet-human bond from a consumer behavior perspective and the lack of knowledge related to the organic food market for pets, specifically in Medellín (Colombia), this quantitative research explores the acceptance of these products in the city. It also seeks to explore the attitudes and purchase intention towards this type of food and its relation with the humanization of pets. The online survey collected 150 responses of consumers between 18 and 65 years of age, belonging to medium and high socioeconomic levels $(4,5$ and 6$)$ in the city. Findings indicate that women have the most positive attitudes towards organic food for pets. More positive attitudes towards this type of food lead to a greater purchase intention. Regarding the level of humanization of pets, there are no significant differences among respondents. The study contributes to the understanding of this type of food market in the capital of Antioquia.
\end{abstract}

Keywords: healthy pets, humanization, human-animal bond, organic food, natural food, attitudes.

a Producciones y Distribuciones Dispro S. A. S., Medellín, Colombia. Correo electrónico: scanogon@eafit.edu.co

b Profesora asociada, Departamento de Marketing, Universidad Eafit, Medellín, Colombia. Correo electrónico: 1ceball4@eafit.edu.co 


\section{Introducción}

El sector de alimentos que ayuda al bienestar y a la salud de las mascotas va en crecimiento. Específicamente, la comida orgánica ha aumentado a nivel mundial, en promedio, un $6.5 \%$ entre 2011 y 2016 (Euromonitor, 2017a). De todos los productos para mascotas, la categoría de alimentos para perros y gatos es la que tiene mayor pronóstico de crecimiento a nivel global. Para 2021, esta categoría va a representar el $70 \%$ del mercado de productos para animales (Euromonitor, 2016). En Latinoamérica dicho mercado ha tenido un crecimiento sostenido entre 2002 y 2014, especialmente en países como Colombia, Perú y Guatemala, donde los mercados aún no están establecidos completamente (Euromonitor, 2017c).

En Colombia el mercado de mascotas entre 2011 y 2016 creció en promedio un 8 \% (Euromonitor, 2017c). Esto también lo confirman datos de la Federación Nacional de Comerciantes (Fenalco), según los cuales una de cada tres familias tiene mascota y existen alrededor de 2 700 establecimientos en el país dedicados a la venta de productos relacionados con estos animales (Ávila, 2016). Adicionalmente, Dinero (2019) aclara que el cambio demográfico hacia más hogares pequeños, unipersonales o sin hijos, ha influido en el aumento de la adquisición de animales de compañía en el país.

Todo esto plantea un mercado potencial e interesante en Colombia, aunque poco explorado y con escasa investigación disponible. Por ejemplo, las grandes multinacionales que dominan el mercado en el país (v. gr., Contegral, Nestlé) no ofrecen aún este tipo de productos en sus portafolios. Sin embargo, este crecimiento no parece una simple moda, pues se está demostrando que este tipo de alimentos en realidad produce beneficios en la salud de las mascotas. Como en el caso de Woodford (2016), quien logró que su perro con cáncer extendiera su vida cuatro años más, después de tener un pronóstico de vida de un año, con una dieta a base de comida orgánica preparada especialmente para su perro.

Debido a las oportunidades que brinda este creciente mercado de alimentos de mascotas, académicos incentivan una mayor investigación para entender el vínculo mascota-humano desde la perspectiva del comportamiento del consumidor (Dotson y Hyatt, 2008). Existe una demanda de estudios que proporcionen información adicional para el cuidado de los seres vivos, especialmente de grupos vulnerables de la sociedad, como los animales de compañía (Hill, Gaines y Wilson, 2008). De igual modo, se requieren trabajos que comprendan el lucrativo segmento de los amantes de las mascotas y las lógicas detrás del comportamiento de consumo de estos (Boya, Dotson y Hyatt, 2015).
Sin embargo, a pesar de la importancia presentada en informes de mercado sobre la humanización de mascotas y los cambios identificados en hábitos de consumo en alimento para mascotas (Euromonitor, 2016), la academia ha tardado en abordar este importante tema. Si bien algunos estudios se han enfocado en cuestiones relativas a la humanización y al consumo de comida para mascotas (Syrjälä y Leipämaa-Leskinen, 2006), estos son limitados, ya que provienen de países desarrollados (v. gr., Finlandia) y, por lo general, no han considerado datos de regiones emergentes como Latinoamérica.

A diferencia del interés de académicos en los alimentos orgánicos para humanos, la exploración sobre alimentos orgánicos para mascotas ha sido escasa. Los trabajos que abordan los alimentos para mascotas se han enfocado, hasta ahora, en: la capacidad de controlar la obesidad en mascotas (Larsen y Villaverde, 2016), el contenido de probióticos (Weese y Arroyo, 2003) y la contaminación de alimentos crudos (Finley, Reid-Smith, Weese y Angulo, 2006), entre otros. La mayoría de estos estudios se han dirigido a los efectos de estos alimentos en la salud de las mascotas, mas no han considerado una aproximación sobre el mercado y el consumo de estos alimentos.

Partiendo de este vacío, y con el fin de reducir esta escasez de información, este estudio cuantitativo aborda el comportamiento del consumidor y tiene como objetivo general el explorar la aceptación de la comida orgánica para mascotas en la ciudad de Medellín, en los estratos o niveles socioeconómicos (NSE) medios-altos (4, 5 y 6). Adicionalmente, el estudio busca conocer la disposición del público objetivo para cambiar la comida tradicional para mascotas por la orgánica, así como identificar si el público objetivo estaría dispuesto a pagar más por comida orgánica para sus mascotas y, por último, medir qué tan humanizado está el mercado de mascotas en Medellín.

El conocimiento generado en este estudio aporta al entendimiento de la relación de los consumidores y sus mascotas, además de presentar los antecedentes sobre la compra de alimentos orgánicos de mascotas. Esto es relevante debido a la magnitud de este negocio que en Colombia moverá 3,5 billones (COP) para 2019 y crecerá un 52,5\% en los siguientes cinco años (Zamora, 2019).

\section{Marco teórico}

La humanización de mascotas y la comida saludable para mascotas

Dentro del mercado de mascotas, la humanización cobra cada vez más una mayor importancia a nivel mundial. En Estados Unidos, según la Asociación Americana de Medicina Veterinaria, el $63 \%$ de los dueños de mascotas las consideran como parte de su familia (AVMA, 2012). 
La tendencia a la humanización, sobre todo de perros y gatos, ha derivado en que los dueños se preocupen más por los ingredientes de la comida para estos (Buff et al., 2014). Por ejemplo, Di Cerbo et al. (2014) hablan sobre cómo el alimentar a las mascotas con dietas parecidas a las humanas ayuda a la prevención de enfermedades, además de mejorar la calidad de vida de las mascotas.

Desde 2016, la tendencia de la humanización de mascotas en Colombia también incrementó la popularidad de productos para su salud y bienestar, provocando una mayor intención de compra de los colombianos hacia este tipo de productos (Euromonitor, 2017b). Tal y como lo expresa el director económico de Fenalco en Colombia, "los animales son considerados parte del núcleo familiar. Por lo cual es cada vez más importante para los colombianos invertir en una buena y sana alimentación para sus mascotas" (Pérez, 2015). No obstante, el interés por el tipo de alimento orgánico y natural se encuentra en sus primeras etapas y se requeriría de esfuerzos para convertirlo en un mercado totalmente aceptado; claro está, con el crecimiento de la humanización esta puede ser una meta alcanzable (Euromonitor, 2017b).

Una de las aproximaciones académicas a la humanización de mascotas va ligada al concepto del "yo extendido" de Belk (1988), en el cual las personas se definen a sí mismas por medio de objetos de consumo, en este caso las mascotas, y a través de estas proyectan sus personalidades y hábitos de consumo. Posteriormente, Belk (1996) y Allen (2003) agregaron que los dueños de mascotas las consideran como parte de sus familias y de sus estilos de vida, lo que hace que también esto se refleje en su parte social y personal, e inviertan grandes cantidades de dinero en ellas.

La humanización de los perros, por ejemplo, es una tendencia creciente que está afectando el comportamiento del consumidor (Boya et al., 2015). Los dueños de perros son cada vez más informados y meticulosos a la hora de comprar alimentos que otorguen beneficios a sus mascotas, esto provoca que la sensibilidad al precio sea cada vez menor (Pask y Scott, 2012). Por tanto, los consumidores están cada vez más dispuestos a pagar significativamente más dinero por comidas premium que sean saludables para sus mascotas (v. gr. orgánica, natural, funcional) (Euromonitor, 2016; Landes, 2010). Por ejemplo, cambios en la dieta de mascotas es un factor influyente en casos de obesidad: enfermedad altamente común en perros y gatos (Larsen y Villaverde, 2016).

Otras investigaciones muestran cómo el desempeño de productos para mascotas está directamente relacionado al vínculo que el dueño tenga con estas (Boya, Dotson y Hyatt, 2012) y que la relación consumidormascota se asemeja a las relaciones humanas (Holbrook et al., 2001). Acero (2017) encontró algunos rasgos de humanización dentro de los colombianos, por ejemplo, cuando se refieren a sus mascotas de manera metafórica (v. gr., mi hijo, mi hermano).

El crecimiento de la tendencia de comida natural para humanos (Barnard, 2010) y la humanización de mascotas han generado el surgimiento del segmento de comida natural para mascotas (Gómez, 2013). Diferentes estudios (Seneviratne et al., 2016; Gómez, 2013; Buff et al., 2014) se apoyan en la definición (traducida del inglés) de alimentos naturales para mascotas propuesta por la Association of American Feed Control Officials (AAFCO, 2012) de Estados Unidos:

Alimento o ingrediente derivado únicamente de fuentes vegetales, animales o minadas, ya sea en su estado no procesado o que haya estado sujeto a procesamiento físico, procesamiento térmico, purificación, extracción, hidrólisis, enzimólisis o fermentación, pero que no haya sido sujeto a un proceso químicamente sintético y que no contenga ningún aditivo de procesamiento que sea quimicamente sintético, excepto en las cantidades que puedan ocurrir en las buenas prácticas de manufactura.

Respecto a la comida orgánica, el Departamento de Agricultura de los Estados Unidos (USDA, 2018) define este tipo de alimentos como aquellos que cumplen con tres requisitos esenciales: 1) no ser realizados con ningún método excluido ( $v$. gr. manipulación genética, radiación), 2) ser producidos con sustancias certificadas y 3) tener la verificación formal de un agente de la USDA. Como ejemplo, la marca Castor \& Pollux ${ }^{1}$, que tiene una mezcla entre comida seca y húmeda para perros y gatos, ofrece un alto nivel de diferenciación al ser de las pocas que cuentan con el sello orgánico de la USDA.

\section{Modelo y desarrollo de hipótesis}

Con el fin de presentar hipótesis relevantes, la figura 1 propone relaciones entre los constructos de humanización de mascotas, actitudes e intención de compra hacia la comida orgánica para mascotas, junto con los antecedentes de edad, género y nivel socioeconómico (NSE). Las lógicas de estas relaciones se plantearán y desarrollarán posteriormente.

\section{Edad}

Según Euromonitor (2014), estudios sobre humanización de mascotas han demostrado que la edad es un factor contradictorio dependiendo del país y la cultura. Por

1 Para más información consultar la página oficial de esta marca: https://www.castorpolluxpet.com. 


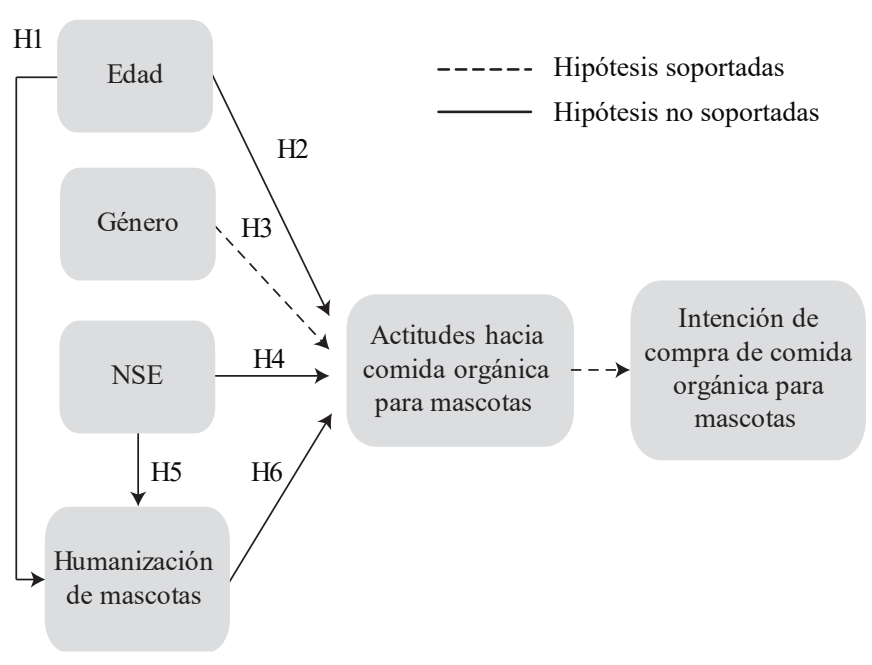

Figura 1. Modelo Propuesto

ejemplo, en países con una cultura hacia mascotas subdesarrollada como China, es común que los jóvenes tiendan a humanizar a su mascota. En países con una cultura más desarrollada hacia las mascotas, como Rusia, es frecuente humanizar cuando se tienen hijos para que estos socialicen. Por último, en una cultura desarrollada como Estados Unidos, la humanización se ve con mayor frecuencia en los adultos mayores que buscan una compañía.

Por otro lado, Zepeda y Li (1991), al igual que Durham (2007), indican que los jóvenes tienen mayor tendencia a preferir la comida orgánica para humanos. Tsakiridou et al. (2008) proponen que las personas mayores son quienes prefieren este tipo de comida, mientras que Lea y Worsley (2005) encontraron que la edad no tenía un impacto en las actitudes hacia la comida orgánica para humanos. Respecto a la edad de los colombianos, la Ley 1885 de marzo de 2018 estipula que una persona joven es aquella que está entre los 14 y los 28 años. Por tanto:

H1: las personas con 28 años o menos tienen mayores niveles de humanización de mascotas que las personas mayores de 29 años.

H2: las personas con 28 años o menos tienen actitudes más positivas hacia la comida orgánica para mascotas que las personas mayores de 29 años.

\section{Género}

Un estudio de Euromonitor (2014) sobre humanización de mascotas concluyó que la mayoría de los dueños de mascotas, un $70 \%$, tiende a humanizar y que, entre estos, es más común en las mujeres que en los hombres. Jones (2013) realizó otro estudio en cuatro países (Australia, Estados Unidos, Japón y Gran Bretaña) y encontró que hay más mujeres que hombres con mascota, también que las mujeres están más relacionadas con actividades que involucren mascotas, sobre todo cuando no tienen hijos, y por tanto tienden a considerar más a sus mascotas como hijos substitutos. Al igual, todo esto se puede relacionar con que las mujeres tienden más a consumir comida orgánica y a preocuparse por la salud de su familia más que los hombres (See Siou Zhen, 2012). Se propone entonces la siguiente lógica:

H3: las mujeres tienen actitudes más positivas hacia la comida orgánica para mascotas que los hombres.

\section{Nivel Socio Económico (NSE)}

Basados en la Ley 142 de 1994, artículo 102, en Colombia los niveles socioeconómicos (NSE) más altos tienen mayores ingresos. Como la comida natural y orgánica para mascotas es considerada como comida premium (Euromonitor, 2017b), se puede deducir que las personas de NSE altos en Colombia tienen mayor tendencia a comprar este tipo de alimentos. Al mismo tiempo, en un mercado con un $70 \%$ de tendencia a la humanización, mayores ingresos significan más dinero para gastar en sus mascotas (Euromonitor, 2014).

Partiendo de una relación entre consumo y humanización, Vänskä (2016) propone que las mascotas son consideradas más queridas por sus dueños, cuando estos están dispuestos a gastar más dinero en ellas. Tesfom y Birch (2010) indican que los dueños de mascotas en Estados Unidos se preocupan más por lo saludable de la comida para sus perros que por la comida para ellos mismos, siendo menos sensibles a los precios de estos alimentos. A partir de este planteamiento se propone:

H4: las personas de NSE más altos tienen actitudes más positivas hacia la comida orgánica para mascotas que las de NSE más bajos.

H5: las personas con NSE más altos tienen mayores niveles de humanización de mascotas que las de NSE más bajos.

\section{Humanización de Mascotas}

Las personas con mayores niveles de humanización se preocupan más por la comida para sus mascotas en términos de qué tan saludable y fresco es el alimento, así como en la calidad y sus valores nutricionales (Boya et $a l .$, 2015). Un estudio realizado por Syrjälä y LeipämaaLeskinen (2006), sobre la relación entre el concepto del "yo extendido" de Belk (1988) y el consumo de comida 
de las mascotas, indica que existe una conexión entre estos dos factores que demuestra el nivel de apego que las personas tienen con las mascotas. Esto genera que en los dueños la parte económica no sea un limitante al momento de comprar la comida de sus animales. Esto lleva a proponer:

H6: las personas con mayores niveles de humanización de mascotas tienen actitudes más positivas hacia la comida orgánica para mascotas que las de menores niveles.

\section{Actitudes}

Según la teoría de actitudes, las actitudes de las personas hacia los objetos (i.e., mascotas) son evaluaciones generales que indican qué tan positiva o negativa es su percepción frente a estos. Si algo genera actitudes positivas en una persona, su intención de compra es generalmente mayor (Ajzen, 1985). El estudio de las actitudes entonces se enfoca principalmente en dos constructos: la actitud y la intención de compra (Spears y Singh, 2004). La teoría de actitudes también establece que estas son las precursoras de los comportamientos, por lo que corrobora la relación altamente estrecha que existe entre la intención y el comportamiento (Aaker, Kumar y Day, 1995; Ajzen y Fishbein, 1977). Esta aproximación es muy apropiada para la presente investigación, ya que al conocer la intención se podrá entonces proyectar el comportamiento.

Los autores Dowd y Burke (2013) identificaron que la teoría de actitudes puede ser aplicada al estudio de la intención y decisión de compra de comida orgánica. E incluso, los consumidores consideran la salud de sus mascotas como un parámetro importante al momento de comprar su alimento (Wandel y Bugge, 1997). Por lo anterior se plantea:

H7: las personas con actitudes más positivas hacia la comida orgánica para sus mascotas tienen mayor intención de compra que las personas con actitudes menos positivas.

\section{Metodología}

La perspectiva ontológica seleccionada es el naturalismo, ya que propone una visión elemental y determinística del fenómeno de estudio (Heath, 1992). Esta investigación se planteó y desarrolló a nivel metodológico a través de la encuesta. Esta técnica cuantitativa de recolección de datos estuvo dirigida a habitantes de barrios de NSE del 4 al 6, de la ciudad de Medellín y el Área Metropolitana, que fueran mayores de 18 años (mayoría de edad en Colombia) y que vivieran con una mascota en su residencia.
Los NSE se clasifican según la Ley 142 de 1994, artículo 102, del 1 al 6; siendo el 4 considerado medio, el 5 como medio-alto y el 6 como alto. Los NSE del 1 al 3 fueron excluidos del estudio debido a que la comida natural y orgánica para mascotas es considerada de calidad superior y tiende a tener un mayor precio de venta (Euromonitor, 2017b). El amplio rango de edad seleccionado se debe a que en muchas ocasiones el dueño de la mascota no elige la alimentación de esta ( $v . g r$. el dueño de la mascota es el hijo de 26 años, pero quien toma decisiones sobre la alimentación es su padre).

Para elegir la población encuestada se usaron las cifras disponibles más recientes, que indican la existencia de alrededor 46000 hogares con perro y 14000 con gato en los NSE 4, 5 y 6 de la ciudad (Alcaldía de Medellín, 2014). Esta cifra permite calcular el potencial mercado que existe para la comida orgánica y natural para perros y gatos en la ciudad. Para calcular la muestra se utilizó un universo de aproximadamente 60000 mascotas, contando con un margen de error del $9 \%$ y un nivel de confianza del $95 \%$. De este modo, la muestra calculada de 119 personas es representativa del sector de la población que toma las decisiones sobre la comida de mascotas (Sapsford, 1999).

\section{Medición}

El cuestionario en Qualtrics (plataforma en línea para crear encuestas) contó en total con seis secciones. La primera, después del consentimiento informado, introdujo preguntas filtro, como: "¿En qué ciudad de Colombia vive?", “Qué especie de mascota tienen en su hogar?”, "Según su cuenta de servicios, ¿cuál es su estrato o nivel socioeconómico?” y “¿Cuál es su edad?”. Si alguna de estas respuestas no coincidía con el perfil buscado, el encuestado era llevado al final de la encuesta. La encuesta continuó indagando características demográficas como género y ocupación. La segunda sección consistió en una escala de 18 ítems tomada de Boya et al. (2015), que comprende los criterios con los cuales los dueños escogen la comida para sus mascotas definiéndose a partir del precio, frescura, calidad, marca, entre otros. Para la clasificación de las respuestas se utilizó una escala Likert de 7 puntos: siendo 1 nada importante y 7 extremadamente importante.

La tercera sección del cuestionario abordó las actitudes de las personas hacia la comida orgánica para mascotas. Para esto se utilizó una escala de cuatro ítems tomados de Yadav y Pathak (2016), como, por ejemplo: "Comprar comida orgánica para mascotas es una buena idea”. En esta sección también se utilizó para las respuestas la escala Likert de 7 puntos de la segunda sección. La cuarta sección hizo referencia a la intención de compra 
de comida orgánica para mascotas, con dos ítems adoptados de Yadav y Pathak (2016), como: "Estoy dispuesto a comprar comida orgánica para mascotas". Para las respuestas se utilizó una escala Likert de 7 puntos: siendo 1 totalmente en desacuerdo y 7 totalmente de acuerdo.

En la quinta sección de la encuesta se indagó sobre la posibilidad de cambiar la comida tradicional para mascotas por la orgánica, por medio de dos preguntas: “¿Estaría usted interesado en comprar comida orgánica para su mascota, en vez de comida tradicional, si tuviese aproximadamente el mismo precio que usted paga actualmente?” y "¿Estaría usted interesado en comprar comida orgánica para su mascota, en vez de comida tradicional, si tuviese un precio mayor al que paga actualmente?". Planteando respuestas en Likert, 7 puntos: siendo 1 totalmente en desacuerdo y 7 totalmente de acuerdo.

La última y sexta sección de la encuesta midió el nivel de humanización de las mascotas por parte de sus dueños, con una escala de 11 ítems, como: "Siento que puedo comunicarme con mi mascota", que fueron adoptados de Boya et al. (2015). Para las respuestas se utilizó una escala Likert de 7 puntos: siendo 1 totalmente en desacuerdo y 7 totalmente de acuerdo. Es importante aclarar que la mayoría de las escalas fueron adaptadas del inglés, para lo cual se realizó una traducción conceptual al español (Dillman, Smyth y Christian, 2009).

\section{Recolección de Datos}

Para el piloto de la encuesta se recolectaron 26 registros. El instrumento fue enviado por medio de un enlace de Qualtrics distribuido por redes sociales. Todas las respuestas fueron útiles para el análisis. Se encontró que el $42.31 \%$ de los participantes tenían perro, $50 \%$ gato y el $7.69 \%$ tenían perro y gato. Respecto al género, el $53.85 \%$ fueron hombres y el $46.15 \%$, mujeres. La distribución del NSE entre 4, 5 y 6 fue de $11.54 \%, 23.08 \%$ y $65.38 \%$, respectivamente. Para el piloto se realizó un análisis de confiabilidad de las escalas utilizadas, por medio del Alfa de Cronbach ( $\alpha$ ), según el cual una escala es confiable si está por encima de .70 (Hair et al., 2013). El resultado arrojó que las escalas en el estudio principal tendrían un buen índice de confiabilidad: actitudes hacia la comida orgánica para mascotas $(\alpha=.97)$, intención de compra de comida orgánica para mascotas $(\alpha=.94)$, y escala de humanización de mascota $(\alpha=.92)$.

La recolección de datos del estudio principal se hizo por medio de un enlace de Qualtrics distribuido a través de redes sociales. Se recolectaron un total de 203 encuestas, de las cuales 150 fueron consideradas útiles para el análisis. Las excluidas correspondían a encuestados que no tienen mascota, que no residen en Medellín y el Área Metropolitana, menores de edad, o que no pertenecen a NSE 4, 5 o 6.
En el análisis de los datos considerados como útiles ( $n=150)$, se encontró que en su mayoría eran mujeres (70.70 \%), pertenecientes al NSE 6 (48.00\%). Las edades estuvieron distribuidas de manera similar, el grupo de 18 a 28 años representa el $46.66 \%$ de los encuestados, mientras que la mayoría de encuestados (53.33\%) reportó edades entre 29 y 65 años. La especie de mascota más encontrada en el análisis fue el perro (61.30 \%), seguido por el gato $(30.70 \%)$, y, por último, ambas mascotas $(8.00 \%)$. El $46.00 \%$ de los participantes indicaron trabajar como empleados.

Se encontró también que dentro de los ítems que más tienen en cuenta los encuestados al elegir la comida para su mascota, están la calidad ( $\mathrm{M}=6.64)$, que sea saludable/nutritivo $(\mathrm{M}=6.54)$ y la recomendación por profesionales en cuidado de la salud de su mascota $(\mathrm{M}=$ 5.89); mientras que los ítems menos importantes son el encontrar información en redes sociales $(\mathrm{M}=2.78)$, que esté en promoción $(\mathrm{M}=2.88)$ y publicidad $(\mathrm{M}=2.97)$, ver tabla 1. Por otro lado, el interés en reemplazar la compra de comida orgánica para mascotas por comida tradicional, fue muy positivo cuando el precio se mantenía $(M=6.09)$ y menor si el precio subía $(M=4.27)$.

Tabla 1. Criterios para elección de comida para mascotas

\begin{tabular}{|c|c|}
\hline Ítem & Media (M) \\
\hline Calidad & 6.64 \\
\hline Saludable / nutritivo & 6.54 \\
\hline $\begin{array}{l}\text { Recomendación por profesionales en cuidado de la salud de } \\
\text { su mascota }\end{array}$ & 5.89 \\
\hline Frescura & 5.82 \\
\hline Facilidad de preparación & 5.70 \\
\hline Sabor & 5.61 \\
\hline $\begin{array}{l}\text { Conveniencia en ubicación de la tienda donde compra la } \\
\text { comida para su mascota }\end{array}$ & 5.13 \\
\hline $\begin{array}{l}\text { Recomendación de expertos en preparación de comida para } \\
\text { mascotas }\end{array}$ & 4.99 \\
\hline Precio & 4.95 \\
\hline Marca & 4.86 \\
\hline Variación en la dieta & 4.49 \\
\hline Recomendación de amigos & 4.18 \\
\hline Holístico/ natural/ orgánico & 4.12 \\
\hline Tipo de tienda donde compra la comida para su mascota & 4.00 \\
\hline $\begin{array}{l}\text { Variedad de productos en la tienda donde compra comida para } \\
\text { su mascota }\end{array}$ & 3.89 \\
\hline Publicidad & 2.97 \\
\hline Está en promoción & 2.88 \\
\hline Redes sociales & 2.78 \\
\hline
\end{tabular}




\section{Resultados y Discusión}

Antes de realizar el testeo de las hipótesis era importante realizar pruebas de confiabilidad (Cronbach $\alpha$ ) para los principales constructos. Los resultados fueron: actitudes hacia la comida orgánica para mascotas $(\alpha=.94)$, intención de compra de comida orgánica para mascotas $(\alpha$ $=.88)$, y humanización de mascotas $(\alpha=.84)$. Posteriormente se verificó por medio de análisis factorial que los principales constructos eran unidimensionales, a excepción de la escala de humanización que reportó que los 11 ítems se agrupaban en dos dimensiones: auto concepto orientado a mascotas, dimensión que alude a "padres" de mascotas que están absorbidos e identificados por sus mascotas y su relación con estas; y antropomorfismo, dimensión que indica el nivel en que los "dueños" de mascotas atribuyen características humanas a sus mascotas y las tratan como "parte de su familia" (Boya et al., 2015, p. 77).

Para el análisis de los datos, las variables de actitudes e intención de compra hacia la comida orgánica y la humanización fueron consideradas como continuas, mientras que edad, NSE y género fueron tratadas como categóricas. Para el testeo de las hipótesis se realizó una prueba T para las H1, H2 y H3 (tablas 2, 3 y 4), Anova para las $\mathrm{H} 4$ y $\mathrm{H} 5$ (tablas 5 y 6 ) y regresiones simples para las $\mathrm{H} 6$ y $\mathrm{H} 7$ (tablas 7 y 8).

Los resultados de la prueba $\mathrm{T}$ para la H1 (tabla 2), mostraron que no hay suficiente evidencia de que la edad influye en el nivel de humanización de las mascotas $\left(\mathrm{M}_{18-28 \text { años }}=4.95\right.$ vs. $\mathrm{M}_{29-65 \text { años }}=4.95$; valor $t=.04$; $p=.97)$; por lo cual H1 no fue soportada. Al mismo tiempo, se encontró por medio de una prueba $\mathrm{T}$ que la edad no influye a la hora de tener actitudes más positivas hacia la comida orgánica para mascotas $\left(\mathrm{M}_{18-28 \text { años }}=\right.$ $5.36 v s . \mathrm{M}_{29-65 \text { años }}=5.41$; valor $t=-.21 ; p=.09$ ), dando por no soportada H2 (tabla 3 ). Mientras que por medio de otra prueba $\mathrm{T}$ (tabla 4) se encontró que sí existe una diferencia significativa de las actitudes hacia la comida orgánica para mascotas por género $\left(\mathrm{M}_{\text {Femenino }}=5.54>\mathrm{M}\right.$ Masculino $=5.00$; valor $t=-1.92 ; p<.001)$, por lo que $\mathrm{H} 3$ fue aceptada.

Tabla 2. Testeo de hipótesis para H1: resultados de la prueba T

\begin{tabular}{c|c|c|c|c|c}
\hline $\mathbf{H}$ & Variable independiente & $\mathbf{n}$ & Media & Valor t & Valor $\boldsymbol{p}$ \\
\hline H1 & $18-28$ años & 70 & 4.95 & .04 & .97 \\
\hline & $29-65$ años & 80 & 4.95 & & \\
\hline
\end{tabular}

Nota: Variable dependiente: Humanización de mascotas.

En relación con $\mathrm{H} 4$ (tabla 5), por medio de un Anova no se encontró evidencia de diferencias significativas de
Tabla 3. Testeo de hipótesis para H2: resultados de la prueba $T$

\begin{tabular}{c|c|c|c|c|c}
\hline $\mathbf{H}$ & Variable independiente & $\mathbf{n}$ & Media & Valor t & Valor $\boldsymbol{p}$ \\
\hline H2 & $18-28$ años & 70 & 5.36 & -.21 & .09 \\
\hline & $29-65$ años & 80 & 5.41 & & \\
\hline
\end{tabular}

Nota: Variable dependiente: Actitudes hacia la comida orgánica para mascotas.

Tabla 4. Testeo de hipótesis para H3: resultados de la prueba T

\begin{tabular}{c|c|c|c|c|c}
\hline $\mathbf{H}$ & Variable independiente & $\mathbf{n}$ & Media & Valor $\boldsymbol{t}$ & Valor $\boldsymbol{p}$ \\
\hline H3 & Masculino & 44 & 5.00 & -1.92 & $.00^{* * *}$ \\
\hline & Femenino & 106 & 5.54 & & \\
\hline
\end{tabular}

Nota: Variable dependiente: Actitudes hacia la comida orgánica para mascotas. $* * * \mathrm{p}<.001$

las actitudes hacia la comida orgánica para mascotas por $\operatorname{NSE}\left(\mathrm{M}_{\mathrm{NSE} 4}=5.42 v s . \mathrm{M}_{\mathrm{NSE} 5}=5.33 v \mathrm{~s} . \mathrm{M}_{\mathrm{NSE} 6}=5.40\right.$; $\left.\mathrm{F}_{(2,149)}=.03, p=.96\right)$, por lo que H4 no es soportada. H5 (tabla 6) indicaba que las personas con NSE más alto tenían mayores niveles de humanización que los que pertenecían a NSE más bajos, pero por medio de un Anova se encontró que no existe tal diferencia $\left(\mathrm{M}_{\mathrm{NSE} 4}=5.06 \mathrm{vs}\right.$. $\mathrm{M}_{\mathrm{NSE} 5}=4.90$ vs. $\left.\mathrm{M}_{\mathrm{NSE} 6}=4.93 ; \mathrm{F}_{(2,149)}=.19, p=.82\right) \mathrm{y}$ H5 fue no soportada.

Tabla 5. Testeo de hipótesis para H4: resultados de Anova

\begin{tabular}{c|c|c|c|c|c}
\hline $\mathbf{H}$ & Variable independiente & $\mathbf{n}$ & Media & Valor $\mathbf{F}$ & Valor $\mathbf{p}$ \\
\hline H4 & NSE 4 & 32 & 5.42 & .03 & .96 \\
\hline & NSE 5 & 46 & 5.33 & & \\
\hline & NSE 6 & 72 & 5.40 & & \\
\hline
\end{tabular}

Nota: Variable dependiente: Actitudes hacia la comida orgánica para mascotas.

Tabla 6. Testeo de hipótesis para H5: resultados de Anova

\begin{tabular}{c|c|c|c|c|c}
\hline $\mathbf{H}$ & Variable independiente & $\mathbf{n}$ & Media & Valor F & Valor $\mathbf{p}$ \\
\hline H5 & NSE 4 & 32 & 5.06 & .19 & .82 \\
\hline & NSE 5 & 46 & 4.90 & & \\
\hline & NSE 6 & 72 & 4.93 & & \\
\hline
\end{tabular}

Nota: Variable dependiente: Humanización de mascotas.

Para H6 (tabla 7) se realizó una regresión simple para encontrar relación entre la humanización y las actitudes hacia la comida orgánica para mascotas, pero los datos hallados no soportaron el planteamiento $(\beta=.20, p=$ $.07)$, por lo cual la H6 no fue soportada. Por último, también se realizó otra regresión simple para probar si había una relación entre las actitudes hacia la comida orgánica para mascotas y la intención de compra, y dicha prueba afirmó tal relación $(\beta=.87, p<.001)$, por lo que $\mathrm{H} 7$ fue 
soportada (tabla 8). El resumen de los resultados presentados de las hipótesis H1-H7 se puede ver en la figura 1.

Tabla 7. Testeo de hipótesis para H6: resultados de regresión simple

\begin{tabular}{c|c|c|c|c|c|c|c}
\hline $\mathrm{H}$ & $\begin{array}{c}\text { Variable } \\
\text { independiente }\end{array}$ & $\mathbf{R}^{2}$ & $\mathbf{B e t a}$ & $\begin{array}{c}\text { Beta } \\
\text { estandarizado }\end{array}$ & $\begin{array}{c}\text { Valor } \\
\boldsymbol{t}\end{array}$ & $\begin{array}{c}\text { Valor } \\
\mathbf{F}\end{array}$ & $\begin{array}{c}\text { Valor } \\
\boldsymbol{p}\end{array}$ \\
\hline $\mathrm{H} 6$ & $\begin{array}{c}\text { Humanización de } \\
\text { mascotas }\end{array}$ & .022 & .20 & .14 & 1.82 & 3.34 & .07 \\
\hline
\end{tabular}

Nota: Variable dependiente: Actitudes hacia la comida orgánica para mascotas.

Tabla 8. Testeo de hipótesis para H7: resultados de regresión simple

\begin{tabular}{c|c|c|c|c|c|c|c}
\hline $\mathbf{H}$ & $\begin{array}{c}\text { Variable } \\
\text { independiente }\end{array}$ & $\mathbf{R}^{2}$ & Beta & $\begin{array}{c}\text { Beta } \\
\text { estandarizado }\end{array}$ & $\begin{array}{c}\text { Valor } \\
\boldsymbol{t}\end{array}$ & $\begin{array}{c}\text { Valor } \\
\mathbf{F}\end{array}$ & $\begin{array}{c}\text { Valor } \\
\boldsymbol{p}\end{array}$ \\
\hline $\mathrm{H7}$ & $\begin{array}{c}\text { Actitudes hacia la } \\
\text { comida orgánica } \\
\text { para mascotas }\end{array}$ & .68 & .87 & .82 & 17.81 & 317.25 & $.00^{* * *}$ \\
\hline
\end{tabular}

Nota: Variable dependiente: Intención de compra de comida orgánica para mascotas.

$* * * \mathrm{p}<.001$

En relación con H1, después de explorar la posible diferencia entre los jóvenes y adultos en Colombia y sus niveles de humanización de mascotas, se encontró que en definitiva tal diferencia no existe. Incluso, los niveles de humanización según la prueba realizada son iguales para ambos grupos de edad $(\mathrm{M}=4.95$, ver tabla 2). Pistas que soporten este resultado se pueden encontrar en Acero (2017), quien indica que la tendencia a la humanización de mascotas en Colombia se da por diferentes motivos y de acuerdo con la edad. Por ejemplo, el autor explica que cuando los jóvenes se casan o se van a vivir juntos es muy común que tengan una mascota con el fin de realizar una especie de "entrenamiento" para luego tener hijos; por lo cual la mascota es tratada por ellos como si fuera un miembro más de la familia.

Por otro lado, Acero (2017) indica que es común que las mascotas se conviertan en parte de la familia de los adultos por dos factores puntuales. El primero, muy relacionado a las amas de casa, quienes están solas durante el día mientras sus esposos trabajan y sus hijos estudian, lo que hace que se cree un vínculo muy grande con su mascota. El segundo, que cuando los hijos dejan de vivir en la casa de sus padres, las mascotas toman ese rol de hijos durante esta etapa.

Los resultados de $\mathrm{H} 2$ indicaron que la edad no está relacionada con actitudes más positivas hacia la comida orgánica para mascotas. Esto es congruente con hallazgos de otras investigaciones sobre comida orgánica para humanos (Durham, 2007; Lea y Worsley, 2005; Tsakiridou et al., 2008; Zepeda y Li, 1991), en los que se encuentran inconsistencias en cuanto a las edades que prefieren en mayor medida este tipo de alimentos. Aunque $\mathrm{H} 2$ no fue significante, este estudio planteó que hay una leve tendencia de los adultos a tener actitudes más positivas hacia la comida orgánica para mascotas que los jóvenes $\left(\mathrm{M}_{18-28 \text { años }}=5.36 v s . \mathrm{M}_{29-65 \text { años }}=5.41\right.$, ver tabla 3$)$.

En cuanto al género, se encontró que las mujeres tienen actitudes más positivas hacia la comida orgánica para mascotas que los hombres, tal y como lo proponía H3. Esto es soportado por Jones (2013) cuando se refiere a las mascotas como hijos sustitutos para las mujeres. Adicionalmente, el resultado de $\mathrm{H} 3$ puede ser explicado por estudios (v. gr. Davies, Titterington y Cochrane, 1995; Fotopoulos y Krystallis, 2002) que indican que las mujeres con hijos se preocupan por la salud de su familia y, por esto, son las que mayor tendencia tienen hacia la comida orgánica para humanos. Por tanto, es probable que las mujeres que ven a sus mascotas como hijos sustitutos y cuidan a su familia con comida orgánica tiendan a su vez a tener actitudes más positivas hacia la comida orgánica para mascotas que los hombres.

Al considerar lo orgánico como comida premium para mascotas, se propuso inicialmente que las personas con mayor poder adquisitivo, reflejado en un mayor NSE, tendrían actitudes más positivas hacia este tipo de comida (H4). Sin embargo, en el análisis de los datos se encontró que esta diferencia no es significativa. Al explorar las razones de este resultado se encontró que precisamente este punto es también ambiguo en la literatura disponible sobre comida orgánica para humanos. Govindasamy e Italia (1999) indicaron que las personas con mayor poder adquisitivo están dispuestas a pagar más por estos productos para humanos; sin embargo, también hay estudios que sostienen que el nivel de ingresos en el caso de la comida orgánica para humanos no es relevante (Yiridoe, Bonti-Ankomah y Martin, 2005).

Es probable que el resultado de $\mathrm{H} 4$ haya sido influenciado por los NSE incluidos en la muestra, porque únicamente se consideraron los NSE del 4 al 6 y se excluyeron los del 1 al 3. Pero curiosamente, en los resultados arrojados por los encuestados de menor NSE son más positivas las actitudes hacia la comida orgánica para mascotas $\left(\mathrm{M}_{\mathrm{NSE} 4}=5.42 v \mathrm{~s} . \mathrm{M}_{\mathrm{NSE} 5}=5.33 v \mathrm{~s} . \mathrm{M}_{\mathrm{NSE} 6}=5.40\right.$, ver tabla 5).

Es evidente que la influencia del NSE sobre la humanización de las mascotas, a partir de H5, no fue significativa. Por tanto, el nivel de ingresos que posiblemente está ligado al nivel de gasto en la mascota, no es lo que hace que se humanice más o menos a estas, sino al contrario. Boya et al. (2015) lo explican al proponer que la humanización por sí sola es lo que hace a las personas más o menos sensibles al precio. En esta investigación, las personas con el NSE más bajo (con menos ingresos) fueron, aunque no de manera concluyente, las que mayores niveles de humanización de mascotas mostraron 
$\left(\mathrm{M}_{\mathrm{NSE} 4}=5.06\right.$ vs. $\mathrm{M}_{\mathrm{NSE} 5}=4.90$ vs. $\mathrm{M}_{\mathrm{NSE} 6}=4.93$, tabla 6). Para un próximo estudio sería ideal explorar todos los NSE, y así poder ver si la humanización de mascotas tiende a aumentar mientras menor sea el NSE.

El nivel de humanización de mascotas no influencia significativamente las actitudes hacia la comida orgánica para mascotas (H6). Para ahondar en los datos que llevan a este resultado, se analizó el coeficiente de correlación de Pearson ( $r$, que indica el grado de relación que existe entre dos variables (Hair et al., 2013). El coeficiente encontrado entre estas dos variables fue: $r$ Humanización de mascotas - Actitudes hacia la comida orgánica para mascotas $=0.15$. Esto indica que hay una relación muy leve entre estas dos variables y que no es significativa en el nivel 0.01 (bilateral), lo que ayuda a soportar que la influencia no sea significativa. Esto puede deberse a que las personas en Colombia, específicamente en Medellín y su Área Metropolitana, tienden a humanizar a sus mascotas, pero esto no necesariamente va relacionado a las actitudes de las personas frente a la comida orgánica para mascotas.

Por último, la influencia de las actitudes hacia la comida orgánica para mascotas sobre la intención de compra de este tipo de alimentos (H7) fue significativa, tal y como la indica la teoría de actitudes (Ajzen y Fishbein, 1977). En definitiva, el perfil de encuestados con actitudes más positivas hacia la comida orgánica para mascotas son mujeres, sin importar la edad, el NSE al que pertenezcan o el nivel de humanización de mascotas que tengan. En cuanto al perfil de los encuestados con mayor nivel de humanización de mascotas, se encontró que no existen diferencias significativas, sin embargo, las mujeres presentan una tendencia mayor $(\mathrm{M}=5.07)$ que los hombres $(\mathrm{M}=4.66)$.

\section{Conclusiones}

Esta investigación cuantitativa buscó explorar la aceptación de la comida orgánica para mascotas en la ciudad de Medellín y su Área Metropolitana. Para esto se enfocó en la humanización de las mascotas, las actitudes de las personas frente a la comida orgánica para mascotas y en comprobar si esto se traducía en intención de compra de este tipo de alimento. Esta arrojó, en términos generales, que tanto las actitudes como la intención de compra hacia la comida orgánica para mascotas son altas, y que son las mujeres quienes presentan actitudes más positivas hacia este tipo de alimentos (H3). Sumado a esto, las actitudes y la intención de compra se encuentran directamente relacionadas (H7). Respecto a la intención de compra, los resultados mostraron que los encuestados estarían altamente de acuerdo en cambiar la comida convencional para mascotas por la orgánica, si esta tuviese idealmente un precio similar al que pagan regularmente.

Por otro lado, los resultados obtenidos mostraron que ni la edad (H2), ni el NSE (H4), ni el nivel de humanización de mascotas (H6), tuvieron significancia en actitudes más positivas hacia la comida orgánica para mascotas. En cuanto a los niveles de humanización de mascotas, y de acuerdo con la clasificación propuesta por Boya et al. (2015), se concluye que los niveles son altos para la ciudad. Sin embargo, estos niveles de humanización no van ligados al poder adquisitivo de las personas, medido por su NSE(H5), ni tampoco existe una relación con la edad (H1).

Como resultado de este estudio, se sugiere que la comida orgánica para mascotas podría tener buena aceptación en la ciudad de Medellín y su área metropolitana, debido a que las actitudes reportadas hacia este tipo de comida son altas, así como la intención de compra. Sin embargo, aunque la humanización es una tendencia mundial, y partiendo de los hallazgos de este estudio, esta no es relevante por ahora para entender el mercado de comida orgánica para mascotas en dicha ciudad. Esta investigación aporta al campo investigativo de la comida orgánica para mascotas y reflexiona sobre las actitudes de las personas frente a este tipo de alimentos, pues existe limitada literatura que conecte ambos, tanto en el mundo como en Colombia.

Como mayor limitante a la investigación se encontró que, si se hubieran incluido todos los NSE, hubiera existido la posibilidad de encontrar significancia en algunas hipótesis. Futuros estudios podrían centrarse en la relación entre la comida orgánica para humanos y para mascotas en Colombia. Además, se podría explorar el tema diferenciando la especie de mascota.

\section{Referencias}

Aaker, D., Kumar, V., \& Day, G. (1995). Attitude measurement. En D. Aaker, V. Kumar \& G. Day (eds.), Marketing Research. (pp. 251-281). Nueva York: Wiley.

Acero, M. (2017). La relación humano-animal de compañía como un fenómeno sociocultural, perspectivas para la salud pública (Tesis doctoral). Universidad Nacional, Bogotá, Colombia. Recuperado de http:// www.bdigital.unal.edu.co/55877/

Alcaldía de Medellín (2014). Encuesta de calidad de vida 2014. Recuperado de https://www.medellin.gov.co

Allen, C. (3 de noviembre de 2003) Owners follow dog's lead when buying pet food. Decision Analyst.

AAFCO (Association of American Feed Control Officials) (2012). Pet Food Regulations. AAFCO Official Publication. Recuperado de https://www.aafco.org/ Regulatory 
AVMA (American Veterinary Medical Association) (2012). Raw or undercooked animal-source protein in cat and dog diets. Recuperado de www.avma.org/KB/ Policies/Pages/Raw-or-Undercooked-Animal-Source-Protein-in-Cat-and-Dog-Diets.aspx

Ajzen, I. (1985). From intentions to actions: a theory of planned behavior. En J. Kuhl \& J. Beckmann (eds.), Action control: From cognition to behavior. (pp. 1139). Nueva York: Springer-Verlag.

Ajzen, I., \& Fishbein, M. (1977). Attitude-behavior relations: A theoretical analysis and review of empirical research. Psychological Bulletin, 84(5), 888-918.

Ávila, R. (2016). Mascotas en Colombia: cariño con millones. Dinero. Recuperado de http://www.dinero. com/opinion/columnistas/articulo/mascotas-en-colombia-carino-con-millones-por-raul-avila/224703

Barnard, N. D. (2010). Trends in food availability, 19092007. The American Journal of Clinical Nutrition, 91(5), 1530-1536.

Belk, R.W. (1988). Possessions and extended self. Journal of Consumer Research, 15,139-168.

Belk, R. W. (1996). Metaphoric relationships with pets. Society and Animals, 4, 120-146.

Boya, U. O., Dotson, M. J., \& Hyatt, E. M. (2012). Dimensions of the dog-human relationship: A segmentation approach. Journal of Targeting, Measurement, and Analysis for Marketing, 20, 133-143.

Boya, U. O., Dotson, M. J., \& Hyatt, E. M. (2015). A comparison of dog food choice criteria across dog owner segments: An exploratory study. International Journal of Consumer Studies, 39(1), 74-82.

Buff, P. R., Carter, R. A., Kersey, J. H., \& Bauer, J. E. (2014). Natural pet food: A review of natural diets and their impact on canine and feline physiology. Journal of Animal Science, 92(9), 3781-3791.

Davies, A., Titterington, A. J., \& Cochrane, C. (1995). Who buys organic food? A profile of the purchasers of organic food in Northern Ireland. British Food Journal, 97(10), 17-23.

Di Cerbo, A., Palmieri, B., Canello, S., Guidetti, G., \& Iannitti, T. (2014). Functional foods in pets and humans. International Journal of Applied Research in Veterinary Medicine, 12(3), 193-200.

Dillman, D. A., Smyth, J. D., \& Christian, L. M. (2009). Internet, mail, and mixed-mode surveys: The tailored design method. Hoboken, NJ: Wiley \& Sons.

Dinero (2019). Crece mercado de mascotas por el cambio demográfico. Recuperado de https://www.dinero. com/empresas/confidencias-on-line/articulo/por-queesta-creciendo-el-mercado-de-mascotas-en-colombia/275308

Dotson, M.J., \& Hyatt, E.M. (2008). Understanding doghuman companionship. Journal of Business Research, 61(5), 457-466.
Dowd, K., \& Burke, K. J. (2013). The influence of ethical values and food choice motivations on intentions to purchase sustainably sourced foods. Appetite, 69, 137-144.

Durham, C. A. (2007). The impact of environmental and health motivations on the organic share of purchases. Agricultural and Resource Economics Review, 36(2), 304-320.

Euromonitor (2014). Pet humanization: The trend and its strategic impact on global pet care markets. Recuperado de http://www.euromonitor.com

Euromonitor (2016). Because they're worth it: pet care global overview. Recuperado de http://www.euromonitor.com/

Euromonitor (2017a, abril). Healthy pets, happy owners: Health and wellness product development in pet care. Recuperado de http://www.euromonitor.com/

Euromonitor (2017b). Pet Food in Colombia. Recuperado de http://www.euromonitor.com

Euromonitor (2017c). Pet Food in Latin America, (April). Recuperado de http://www.euromonitor.com/

Finley, R., Reid-Smith, R., Weese, J. S., \& Angulo, F. J. (2006). Human health implications of Salmonellacontaminated natural pet treats and raw pet food. Clinical Infectious Diseases, 42(5), 686-691.

Fotopoulos, C., \& Krystallis, A. (2002). Purchasing motives and profile of the Greek organic consumer: A countrywide survey. British Food Journal, 104(9), 730-765.

Gómez, L. M. (2013). Introducción a la nutrición de caninos y felinos. Journal of Agriculture and Animal Sciences, 2(2), 52-67.

Govindasamy, R. e Italia, J. (1999). Predicting willingness-to-pay a premium for organically grown fresh produce. Journal of Food Distribution Research, 30(2), 44-53.

Hair, J. F., Black W. C., Babin B. J., \& R. E. Anderson. (2013). Multivariate data analysis: A global perspective. Noida: Pearson.

Heath, T. B. (1992). The reconciliation of humanism and positivism in the practice of consumer research: A view from the trenches. Journal of the Academy of Marketing Science, 20(2), 107-118.

Hill, R. P., Gaines, J., \& Wilson, R. M. (2008). Consumer behavior, extended self, and sacred consumption: An alternative perspective from our animal companions. Journal of Business Research, 61(5), 553-562.

Holbrook, M., Stephens, D. L., Day, E., Holbrook, S., \& Strazar, G. (2001). A collective stereographic photo essay on key aspects of animal companionship: The truth about cats and dogs. Academy of Marketing Science Review, 1(1), 1-17.

Jones K. (2013). Sexual differences in psychology and pet ownership. Recuperado de http://www.acac.org. au/pdf/PetFactBook_June 
Landes, L. (2010) Pet ownership: A financial and emotional responsibility. Consumerism Commentary. Recuperado de http://www.consumerismcommentary.com

Larsen, J. A., \& Villaverde, C. (2016). Scope of the problem and perception by owners and veterinarians. Veterinary Clinics of North America-Small Animal Practice, 46(5), 761-772.

Lea, E., \& Worsley, T. (2005). Australians' organic food beliefs, demographics and values. British Food Journal, 107(11), 855-869.

Pask, E., \& Scott, L. (2012). Feeding fido: how to really read a dog food label. Modern Dog Magazine. Recuperado de http://moderndogmagazine.com/ articles/ feeding-fido/4870

Pérez, V. (2015). ¿Qué concentrados prefieren las mascotas? La República. Recuperado de https://www.larepublica.co/archivo/que-concentrados-prefieren-lasmascotas-2223866

Real Academia Española (2014). Diccionario de la lengua española (23 ed.). Recuperado de http://dle.rae. es/?id=KnX9kjd

Sapsford, R. (1999). Survey research. Londres: Sage.

See Siou Zhen, J. (2012). Young female motivations for purchase of organic food in Malaysia. International Journal of Contemporary Business Studies, 5(3), 6172. Recuperado de http://www.akpinsight.webs.com

Spears, N., \& Singh, S. N. (2004). Measuring attitude toward the brand and purchase intentions. Journal of Current Issues \& Research in Advertising, 26(2), 5366.

Seneviratne, M., Subasinghe, D. W. D., \& Watson, P. J. (2016). A survey of pet feeding practices of dog owners visiting a veterinary practice in Colombo, Sri Lanka. Veterinary Medicine and Science, 2(2), 106116.

Syrjälä, H., \& Leipämaa-Leskinen, H. (2006). Pets as extended self in the context of pet food consumption. European Advances in Consumer Research, 7, 543-549.
Tesfom, G., \& Birch, N. (2010). Do they buy for their dogs the way they buy for themselves? Psychology and Marketing, 27(9), 898-912.

Tsakiridou, E., Boutsouki, C., Zotos, Y., \& Mattas, K. (2008). Attitudes and behaviour towards organic products: An exploratory study. International Journal of Retail \& Distribution Management, 36(2), 158-175.

USDA (2018). Organic Regulation. Recuperado de https://www.ams.usda.gov/rules-regulations/organic

Vänskä, A. (2016). “Cause I wuv you!” Pet dog fashion and emotional consumption. Ephemera, 16(4), 75-97.

Wandel, M., \& Bugge, A. (1997). Environmental concern in consumer evaluation of food quality. Food Quality and Preference, 8(1), 19-26.

Weese, J. S., \& Arroyo, L. (2003). Bacteriological evaluation of $\mathrm{dog}$ and cat diets that claim to contain probiotics. The Canadian Veterinary Journal, 44(3), 212.

Woodford, R. (2016). "People food" for dogs. Better Nutrition, 78(7), 26-27.

Yadav, R., \& Pathak, G.S. (2016). Intention to purchase organic food among young consumers: Evidences from a developing nation. Appetite, 96, 122-128.

Yiridoe, E. K., Bonti-Ankomah, S., \& Martin, R. (2005). Comparison of consumer perceptions and preference toward organic versus conventionally produced foods: A review and update of the literature. Renewable Agriculture and Food Systems, 20(4), 193-205.

Zamora, H. F. (11 de agosto de 2019). Mascotas, negocio que movería $\$ 3.5$ billones este año. Portafolio. Recuperado de: https://www.portafolio.co/negocios/ mascotas-negocio-que-moveria-3-5-billones-esteano-532462

Zepeda, L. \& Li, J. (2007). Characteristics of organic food shoppers. Journal of Agricultural and Applied Economics, 29(1), 17-28.

Yañez, R., Arenas, M., \& Ripoll, M. (2010). El impacto de las relaciones interpersonales en la satisfacción laboral general. Revista de Psicología, 16(2), 193-201. 


\section{Anexo}

Tabla A. Características de la muestra $(n=150)$

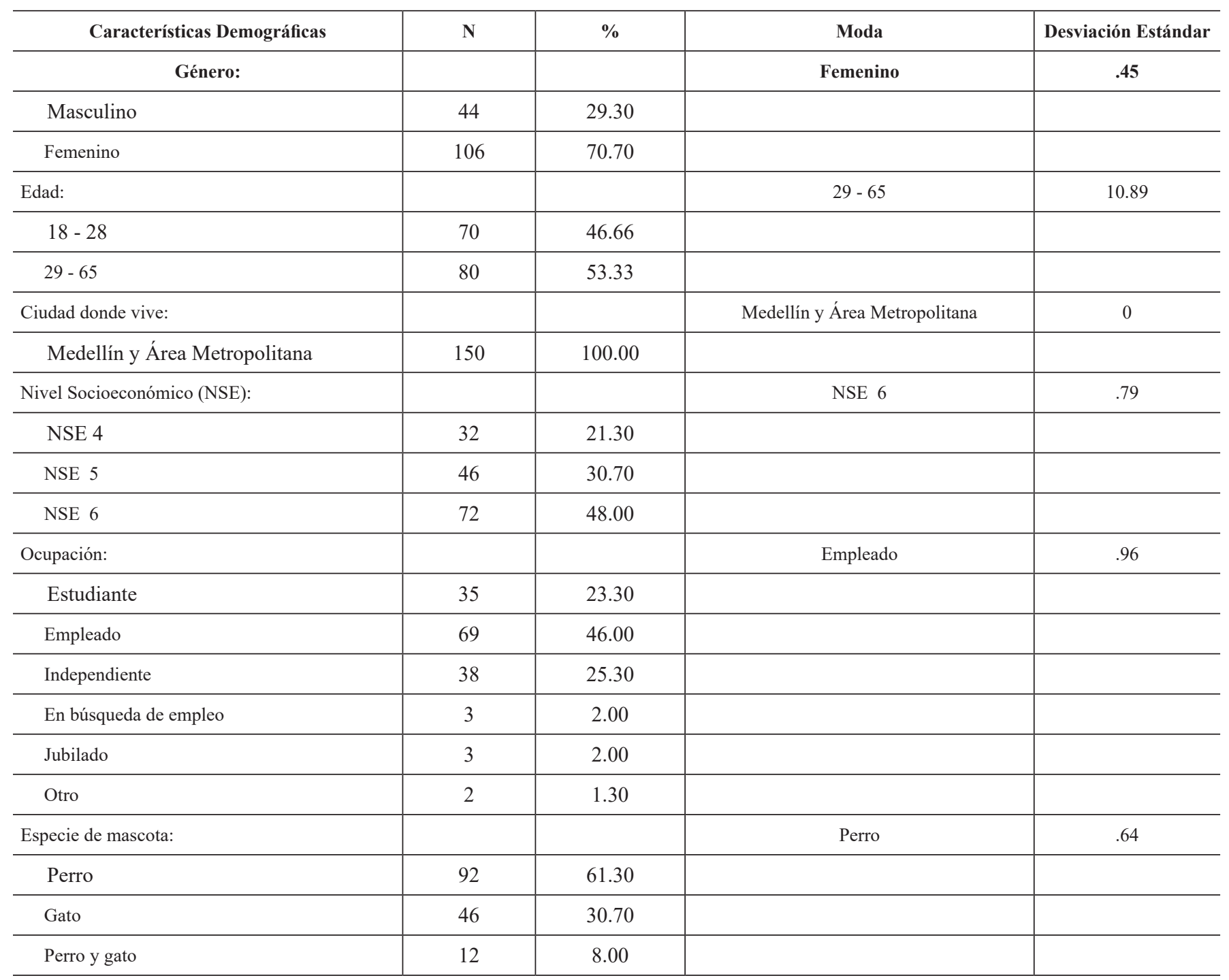

